Research Article

\title{
What Makes Us Walk: Predictors and the Interplay of Physical and Cognitive Factors on Gait Speed in Community Dwelling Older Adults
}

\author{
Ashleigh Trapuzzano, Sara Chizmar, Lauren Wilda, Nicole Dawson *
}

University of Central Florida, Doctor of Physical Therapy Program, School of Kinesiology and Physical Therapy, Orlando, FL, USA; E-Mails: atrapuzzano29@gmail.com; smchizmar@knights.ucf.edu; lauren.haffke@knights.ucf.edu; nicole.dawson@ucf.edu

* Correspondence: Nicole Dawson; E-Mail: nicole.dawson@ucf.edu

Academic Editor: James S. Powers

Special Issue: Geriatric Syndromes

OBM Geriatrics

2020, volume 4, issue 3

doi:10.21926/obm.geriatr.2003134
Received: April 03, 2020

Accepted: September 23, 2020

Published: September 27, 2020

\begin{abstract}
This study investigated the predictors of gait speed in community dwelling older adults while examining interplay between physical performance and cognition on comfortable and fast gait speed. Sixty-six community-dwelling older adults (mean age $80.871 \%$ female) completed the following: 30-Second Chair Stand (30-SCS), Functional Reach (FR), Flanker Task, Digit Symbol Substitution Test (DSST), and gait speed (comfortable and fast). Hierarchical linear regression examined the relationship of comfortable and fast gait speeds with physical performance (30-SCS, FR) and cognitive domains (DSST, CDT, Flanker effect). Unique predictors of comfortable gait speed included 30-SCS ( $B=1.86, p<0.001), F R(B=3.37$, $p=0.005)$, and Flanker effect $(B=-0.02, p=0.05)$. Unique predictors of fast gait speed included 30-SCS $(B=2.61, p<0.001), F R(B=3.58, p=0.04)$, and DSST $(B=0.95, p=0.01)$. Both comfortable and fast gait speed were primarily predicted by strength and balance while cognitive factors, including executive function and processing speed, also contribute to predicting gait speed. Lower extremity strength and balance are independently predictive of both comfortable and fast gait speed. Executive function and cognitive inhibition, as assessed by the Flanker effect, predicted comfortable gait speed, while processing speed, as assessed by the DSST,
\end{abstract}

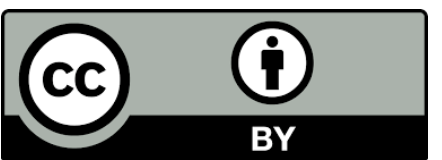

(c) 2020 by the author. This is an open access article distributed under the conditions of the Creative Commons by Attribution License, which permits unrestricted use, distribution, and reproduction in any medium or format, provided the original work is correctly cited. 
predicted fast gait speed. These results corroborate previous literature that examined functional and cognitive domains individually.

\section{Keywords}

Functional outcomes; gait speed; rehabilitation; cognition

\section{Introduction}

Adults aged sixty or older numbered an estimated 962 million worldwide in 2017, with the expectation that this number will double by 2050 [1]. In the United States alone, adults aged 65 and or older comprised $15.2 \%$ of the population in 2016, numbering 49.2 million [2]. To optimize health and function in this growing population, clinicians and researchers alike must understand the most influential factors on future health and wellness. Additionally, it is important to determine inexpensive and efficient outcomes measures that can identify decline in those factors which are amenable to intervention. Gait speed is one such factor that is both easily measurable and has established reliability and minimum detectable change values [3, 4]. These strong psychometric properties and versatile clinical utility have contributed to gait speed being considered "the 6th vital sign" [5]. It is recognized as a valid tool that has demonstrated predictive ability for both functional mobility and overall health status [3]. Gait speed has been identified as a key indicator in other important health outcomes, such as mortality, institutionalization, and dependence in daily activities; therefore, it crucial to understand the factors that may influence an older adults' gait speed [5].

Mobility through ambulation is required for independence with activities of daily living and participation in community activities. Further, older adults must be able to vary their gait speed to successfully and safely complete functional tasks such as hurrying to the rest room or crossing a busy street. In a healthy population of older adults, those individuals ambulating at a speed below a cut off of 1.0 meters per second have been found to be at higher risk for health related outcomes including lower extremity limitations, hospitalization, and death [6]. Faster gait speeds overall have been found to be associated with higher levels of independence and survival in older adults $[7,8]$. Both comfortable and fast gait speeds have demonstrated prognostic value for identifying health related outcomes such as disability $[9,10]$, falls $[11,12]$, cognitive decline [13], and mortality [11].

Current literature recognizes a number of influencing variables on gait speed including balance [14-17], lower extremity muscle strength [14, 15, 18, 19], cognition [20-22], and demographic characteristics (e.g. age) [17]. In particular, age related decreases in strength have a substantial impact on physical performance including gait speed. With primary aging, type II muscle fibers decrease with an additional reduction in motor unit innervation [23]. Lean muscle mass decreases as fat mass increases resulting in sarcopenia [24]. As a result, there is an overall reduction in muscle strength, leading to potential functional mobility limitations and a higher risk for falls and hospitalizations [25]. Multiple studies have identified both composite lower extremity muscle strength and individual muscle strength as predictive variables for comfortable and fast gait speeds [14, 18, 26-28]. Aranda-Garcia (2015) found that isometric knee extensor strength was the 
best predictor of fast gait speed in community dwelling older adults, explaining $47.5 \%$ of the variance in both global characteristics and physical abilities models. Similarly, another study reported that isometric knee extensor strength explained $36 \%$ of the variance of comfortable gait speed among a sample of 839 older adults [18]. Balance has also been shown to influence gait speed through assessment of various components of standing balance [14-17].

Another variable to consider during evaluation of the older adult is cognitive function and its impact on physical abilities. Gait requires cognitive integration of motor commands and perceptual sensory inputs to execute a normal gait cycle [29]. Daily life often requires older adults to adapt to environmental demands while walking and, under these circumstances, cognitive resources are in higher demand [29]. Callisaya and colleagues (2017) found that older adults with higher levels of cognitive impairment had slower comfortable and fast gait speeds and poorer ability to increase gait speed. Determining the most important cognitive domains related to gait speed is essential to development of cognitive interventions for mobility decline and disability. Domains of executive function and processing speed have been associated with gait parameters, including gait speed [20-22, 30,31]. Executive function includes multiple processes including attention, planning, organizing, inhibition, and directing goal-oriented behavior. Processing speed is the time to perceive, process, and direct cognitive information. Martin et al. (2012) found that executive function and processing speed were independently associated with comfortable gait speed along with other associated gait parameters. Another study found that measures of executive function, processing speed, memory, and verbal IQ explained $16 \%$ of the variance of comfortable gait speed among 186 community-dwelling older adults [32]. Further, it has been reported that a faster reaction time is associated with increased comfortable gait speed with the authors postulating that a slower reaction time may be related to reduced central processing speed [17] To add to these findings, numerous longitudinal studies have reported that lower baseline scores on tests of executive function, processing speed, and memory led to faster yearly decline in walking speed [30, 33, 34]. Although these relationships are strong, few studies have included other measures of physical function in their analyses to determine if there is shared variance between cognitive and physical variables.

The current literature offers some insight into the role of physical and cognitive factors on predicting gait speed, however a gap in the literature is present in regard to the interplay between these factors. Very few studies include both physical performance and cognitive measures in the same analyses. Understanding if shared variance exists between strength, balance, and specific cognitive domains can guide clinical treatment when gait speed deficits are present. Further, many studies investigating the influence of cognition have focused on comfortable gait speed, but not fast gait speed. It is important to determine if both comfortable and fast gait speed are influenced by the same factors or if they should be considered separately during the evaluation and treatment of older adults as prior studies have found differences in predictor variables (Mantel et al., 2018). Therefore, the aim of the current study was to investigate the unique predictors of both comfortable and fast gait speed in community dwelling older adults using measures of cognition and physical performance. Two hypotheses were made: 1) lower extremity strength and balance would be strong predictors of both comfortable and fast gait speed with statistical control of cognitive variables and (2) executive function would be the strongest cognitive predictor of both comfortable and fast gait speed. 


\section{Methods}

\subsection{Participants and Study Design}

Participants included 66 community-dwelling older adults recruited from two retirement communities in Florida. A sample size of 66 was determined based on a power of 0.80 , an alpha level of $\leq 0.05$ with a medium to large effect size as demonstrated by previous literature regarding the predictors of gait speed, and use of four to six independent variables in a multiple linear regression model [35]. Participants were initially screened through a telephone interview to determine eligibility based on the following inclusion criteria: 60 years of age or older and able to walk at least 20 feet without an assistive device. Participants provided verbal informed consent prior to participation.

\subsection{Procedure}

This observational study was composed of a one-time data collection scheduled individually for each participant. Upon receiving verbal informed consent, each participant completed a short demographic and health questionnaire. Testing was completed at a central location within the retirement community by the same researcher. Physical performance-based measures included the 30-Second Chair Stand test (30-SCS) [36] and the Functional Reach test (FR) [37]. These tests were selected because they are reliable and valid tests that are easy to perform in the clinic. Cognitive assessments included the Mini-Mental State Examination as a measure of global cognition, the Clock-drawing test (CDT) [38], Trail Making Test Part B (TMT-B) [39], Flanker Compatibility Task [40], simple reaction time test, and the Digit Symbol Substitution Test (DSST) [41]. All cognitive assessments were performed on an iPad with the exception of the TMT-B and DSST, which were completed using a paper format. Physical performance and cognitive assessments were completed together in one block in the order as described above, however, to protect against order effects, the protocol was counterbalanced to have some participants performed the physical measures first while others performed the cognitive measures first.

\subsection{Gait Assessment}

Gait speed was assessed with the 12 -foot GAITRite ${ }^{\circledR}$ system, an electronic walkway designed to assess spatiotemporal gait parameters [42]. To allow for acceleration before stepping on the walkway, participants were instructed to take two steps away from the start of the walkway before each trial and then center their body facing the walkway. Participants were asked to walk at a "comfortable pace" on the GAITRite ${ }^{\circledR}$ for two trials and then were asked to walk "as fast as you can while staying safe" for two trials. Gait velocity $(\mathrm{cm} / \mathrm{s})$ was recorded for each trial. The average of the two trials (comfortable and fast) was used in statistical analyses.

\subsection{Physical Performance Measures}

The 30-second Chair Stand Test (30-SCS) assessed lower-extremity strength by counting the number of full stands from a standard 17-inch chair without use of arms from a seated position in 30 seconds [36]. If a participant was unable to complete a single repetition without the use of hands, a score of zero was recorded. To assess standing balance, the Functional Reach (FR) test 
was performed [37]. Participants were instructed to stand close to the wall without touching it and position their arm at 90 degrees of shoulder flexion with a closed fist. Participants were then instructed to "Reach as far as you can forward without taking a step". The location of the 3rd metacarpal was recorded from zero on a yardstick to the end point after reaching forward[37].

\subsection{Cognitive Measures}

The Clock-drawing test was used to assess executive function and visuospatial function [38]. Participants were presented with a pre-drawn blank circle and asked to "draw the numbers on the circle to make it look like a clock and draw the hands to read 10 past 11 o'clock". The scoring method described by Watson and colleagues was used with a normal score ranging from 0-3 and abnormal score ranging from 4-7 [43]. The TMT-B was used to assess executive function and setshifting [39]. Participants were instructed to connect 25 circles in an ascending pattern, without lifting the pencil, with the task of alternating between numbers and letters (numbers 1-13; letters $A-L)$. Scores were reported as the number of seconds to complete the task, with higher scores representing greater cognitive impairment [39].

The Flanker Compatibility Task was used to assess executive function, cognitive inhibition, and selective visual attention [40]. This test was administered using the PsychLab101 iPad app [44]. Participants were instructed to decide whether they saw a square or a diamond within a display of four rings presented on the screen while ignoring any other shapes that appeared to the side of the array of rings (see Figure 1). Test trials were either congruent (the stimulus matched the stimulus to the side of the rings), incongruent (the stimulus did not match the stimulus to the side of the rings), or neither (no distractor stimulus appeared). The Flanker effect has been described as the effect of the conflict resolution on performance, such that congruent stimuli produce faster and more accurate responses than incongruent stimuli [45] In this study, the Flanker effect was calculated as the difference in mean reaction time between congruent and incongruent trials [45].

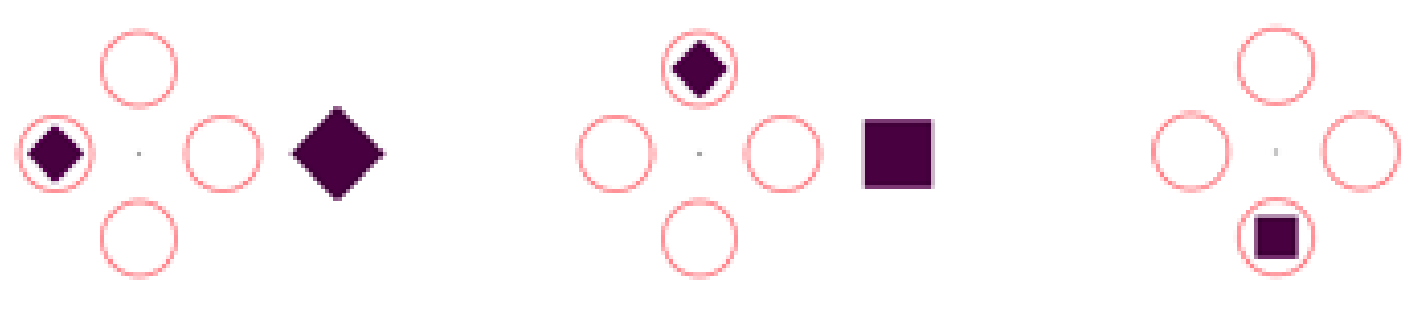

Figure 1 Flanker compatibility task (Neurobehavioral Systems, 2015). Used to assess executive function, cognitive inhibition, and selective visual attention and administered using the PsychLab101 iPad app (Neurobehavioral Systems, Inc., Berkeley CA) [38-40]. Participants were instructed to identify if the target stimulus in the rings was a square or a diamond, while ignoring distractor stimuli outside the rings. Test trials included distractor stimuli that were either congruent (right), incongruent (middle), or neither (left). 
A simple reaction time test was also administered using the PsychLab101 iPad app [44] Participants were instructed to touch the iPad screen as fast as possible when they saw the stimulus appear on the screen. The score was recorded as the average reaction time (milliseconds) for the total number of trials given. Lastly, the Digit Symbol Substitution Test was used to assess processing speed [41]. Participants matched a given number (1-9) to its corresponding symbol using the key grid at the top of the testing paper (see Figure 2). Participants were instructed to fill in as many boxes as possible in 90 seconds, in the order that they appeared. The number of boxes completed (maximum $=90$ ) were recorded.

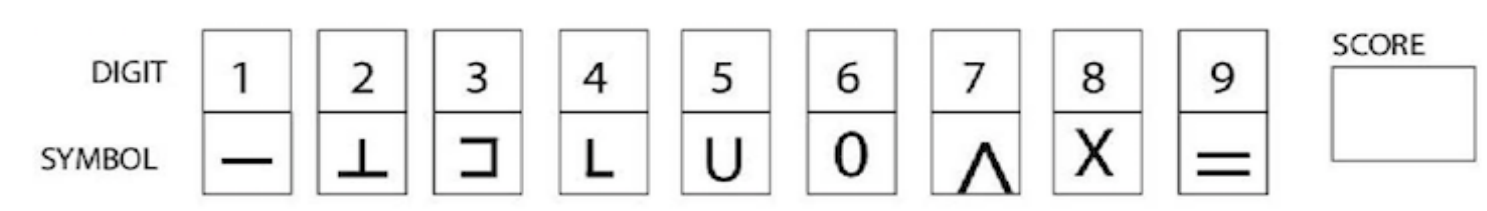

\begin{tabular}{l}
\hline \hline \multicolumn{110}{c|}{} \\
\begin{tabular}{|l|l|l|l|l|l|l|l|l|l|l|l|l|l|l|l|l|l|l|l|l|l|l|l|l|}
\hline 2 & 1 & 3 & 7 & 2 & 4 & 8 & 1 & 5 & 4 & 2 & 1 & 3 & 2 & 1 & 4 & 2 & 3 & 5 & 2 & 3 & 1 & 4 & 6 & 3 \\
\hline & & & & & & & & & & & & & & & & & & & & & & & & \\
\hline
\end{tabular}
\end{tabular}

\begin{tabular}{|l|l|l|l|l|l|l|l|l|l|l|l|l|l|l|l|l|l|l|l|l|l|l|l|l|}
\hline 1 & 5 & 4 & 2 & 7 & 6 & 3 & 5 & 7 & 2 & 8 & 5 & 4 & 6 & 3 & 7 & 2 & 8 & 1 & 9 & 5 & 8 & 4 & 7 & 3 \\
\hline & & & & & & & & & & & & & & & & & & & & & & & & \\
\hline
\end{tabular}

\begin{tabular}{|l|l|l|l|l|l|l|l|l|l|l|l|l|l|l|l|l|l|l|l|l|l|l|l|l|}
\hline 6 & 2 & 5 & 1 & 9 & 2 & 8 & 3 & 7 & 4 & 6 & 5 & 9 & 4 & 8 & 3 & 7 & 2 & 6 & 1 & 5 & 4 & 6 & 3 & 7 \\
\hline & & & & & & & & & & & & & & & & & & & & & & & & \\
\hline
\end{tabular}

\begin{tabular}{|l|l|l|l|l|l|l|l|l|l|l|l|l|l|l|l|l|l|l|l|l|l|l|l|l|}
\hline 9 & 2 & 8 & 1 & 7 & 9 & 4 & 6 & 8 & 5 & 9 & 7 & 1 & 8 & 5 & 2 & 9 & 4 & 8 & 6 & 3 & 7 & 9 & 8 & 6 \\
\hline & & & & & & & & & & & & & & & & & & & & & & & & \\
\hline
\end{tabular}

Figure 2 Digit symbol substitution test [41]. Used to assess processing speed [41]. Following practice of the sample boxes, participants were instructed to match the correct corresponding symbol into the numbered boxes in the order that they are presented below. The number of boxes completed in 90 seconds was recorded.

\subsection{Statistical Analysis}

Data were input into SPSS Statistical Software (Version 22.0, IBM Statistics) for analysis. Following preparatory analyses, descriptive statistics were used for sample representation and comparison to known normative data. Bivariate correlation (Pearson's $r$ ) analyses were examined to determine the level of association between the dependent variables of comfortable and fast gait speed and the independent variables of physical performance and cognition. Bivariate correlation was used to assist in determination of which independent variables would be included in the regression analyses.

The results from these analyses determined the independent variables used in the hierarchical linear regression models. Hierarchical linear regression models were constructed to examine the independent association of both comfortable and fast gait speeds with physical performance (30- 
SCS and FR) and cognition (DSST, CDT, and Flanker effect). Also considered was the concern of multicollinearity between variables as it may inflate the size of the error terms, which could weaken the analysis by making it more difficult to reject the null hypothesis [46]. Subsequently, variables with bivariate correlations of greater than .70 should only be cautiously entered into the same analysis as these variables may be found to have multicollinearity [47] Therefore due to the high correlation between the TMT-B and DSST $(r=0.70)$, the TMT-B was not included in regression model. In Model 1, the physical performance variables were included followed by the cognitive variables in Model 2. This approach allows separate analysis regarding contribution of each set of variables (physical performance variables and cognitive variables) to the prediction of gait speed. Alpha level was set at .05.

\section{Results}

The participant characteristics are presented in Table 1 . The average age of the participants was 80.80 years (SD: 8.01 ) and $71 \%$ were female. The participants in the study had an average comfortable gait speed of $1.00 \mathrm{~m} / \mathrm{s}$ (SD: 0.27 ) and an average fast gait speed of $1.41 \mathrm{~m} / \mathrm{s}$ (SD: 0.37).

Bivariate correlation coefficients are reported in Table 2. Comfortable gait speed was significantly associated with the 30-SCS $(r=0.54 ; p=<0.001)$, FR $(r=0.53 ; p=<0.001)$, TMT-B $(r=$ $-0.34 ; p=0.004)$, total number of boxes completed on the DSST $(r=0.48 ; p=<0.001)$, and flanker effect $(r=-0.28 ; p=0.02)$. There was no significant association with simple reaction time; therefore, it was not included in further analyses. Fast gait speed was significantly associated with the 30-SCS $(r=0.55 ; p=<0.001)$, FR $(r=0.44 ; p=<0.001)$, TMT-B $(r=-0.32 ; p=0.007)$, total number of boxes completed on the DSST $(r=0.57 ; p=<0.001)$, and CDT $(r=-0.26 ; p=0.03)$. No significant association was found between fast gait speed and simple reaction time; therefore, it was not analyzed further. In the hierarchical linear regression (Tables 3 and 4), physical performance variables including the 30 -SCS and FR explained $44.80 \%$ and $38.20 \%$ of the variance (adjusted $r^{2}$ ) in comfortable and fast gait speed, respectively as outlined in Model 1 . The addition of cognition in Model 2 contributed significantly to both comfortable ( $F$ change $=3.34 ; p=0.04$ ) and fast ( $F$ change $=3.78 ; p=0.03$ ) gait speed. Unique predictors of comfortable gait speed in Model 2 included 30-SCS $(B=1.86, p<0.001), F R(B=3.37, p=0.005)$, and Flanker effect $(B=-0.02$, $p=0.05)$. This indicates that individuals who demonstrated greater lower extremity strength and balance and demonstrated greater executive function and cognitive inhibition were able to walk at a faster pace in the comfortable gait speed condition. Unique predictors of fast gait speed in Model 2 included 30-SCS $(B=2.61, p<0.001)$, FR ( $B=3.58, p=0.04)$, and DSST $(B=0.95, p=0.01)$. This indicates that individuals who demonstrated greater lower extremity strength and balance and demonstrated greater processing speed were able to walk at a faster pace in the fast gait speed condition. 
Table 1 Participant demographic characteristics $(N=66)$.

\begin{tabular}{|c|c|c|c|}
\hline Variable & Mean (SD) & Range & Reference, normal \\
\hline Age, y & $80.8(8.01)$ & $61-86$ & \\
\hline $\begin{array}{l}\text { Comorbid Health } \\
\text { Conditions }\end{array}$ & $3.18(1.74)$ & $0-8$ & \\
\hline Falls in Past Month & $0.22(0.54)$ & $0-3$ & \\
\hline $\begin{array}{l}\text { Mini-Mental Status } \\
\text { Examination Score }\end{array}$ & $28.57(1.31)$ & $25-30$ & $\begin{array}{c}<24 / 30, \text { considered } \\
\text { abnormal }\end{array}$ \\
\hline $\begin{array}{l}\text { 30-Second Chair Stand } \\
\text { Score, repetitions }\end{array}$ & $10.38(6.0)$ & $0-25$ & 9-14 repetitions \\
\hline Functional Reach Score, in & $9.87(2.32)$ & $5.08-15.17$ & 10.5 inches \\
\hline $\begin{array}{l}\text { Comfortable Gait Speed, } \\
\mathrm{m} / \mathrm{s}\end{array}$ & $1.0(0.27)$ & $0.32-1.55$ & $0.85-1.03 \mathrm{~m} / \mathrm{s}$ \\
\hline Fast Gait Speed, cm/s & $1.41(0.37)$ & $0.46-2.30$ & $1.59 \mathrm{~m} / \mathrm{s}$ \\
\hline Clock Drawing Test score & $1.97(2.46)$ & $0-7$ & $>4$, considered abnormal \\
\hline $\begin{array}{l}\text { Flanker Task Reaction Time, } \\
\text { Congruent Trials }\end{array}$ & $1597.53(550.13)$ & $1002.3-4331.0$ & \\
\hline $\begin{array}{l}\text { Flanker Task Reaction Time, } \\
\text { Incongruent Trials }\end{array}$ & $1701.43(477.72)$ & $953.31-3458.2$ & \\
\hline $\begin{array}{l}\text { Trail Making Test (Part B), } \\
\text { time in seconds }\end{array}$ & $127.45(81.22)$ & $11.31-519.00$ & 90 seconds \\
\hline $\begin{array}{l}\text { Digit Symbol Substitution } \\
\text { Test, number completed }\end{array}$ & $42.09(12.5)$ & $13-76$ & \\
\hline
\end{tabular}

Table 2 Pearson correlation matrix.

\begin{tabular}{|l|c|c|}
\hline \multicolumn{1}{|c|}{ Variable } & $\begin{array}{c}\text { Comfortable Gait Speed, } \\
\mathrm{cm} / \mathrm{s}\end{array}$ & Fast Gait Speed, cm/s \\
\hline 30-Second Chair Stand Score & $0.54^{\mathrm{a}}$ & $0.55^{\mathrm{a}}$ \\
\hline Functional Reach Score, in & $0.53^{\mathrm{a}}$ & $0.44^{\mathrm{a}}$ \\
\hline Trail-Making Test (Part B) & $-0.34^{\mathrm{a}}$ & $-0.32^{\mathrm{a}}$ \\
\hline Clock Drawing Test score & -0.20 & $-0.26^{\mathrm{b}}$ \\
\hline Flanker Effect & $-0.28^{\mathrm{b}}$ & -0.22 \\
\hline $\begin{array}{l}\text { Digit Symbol Substitution Test, } \\
\text { number completed }\end{array}$ & $0.48^{\mathrm{a}}$ & $0.52^{\mathrm{a}}$ \\
\hline
\end{tabular}

Notes: ${ }^{a} p<.001 ;{ }^{b} p<.05$ 
Table 3 Hierarchical linear regression summary for comfortable gait speed $(\mathrm{cm} / \mathrm{s})$.

\begin{tabular}{|c|c|c|c|c|c|c|}
\hline \multirow[b]{2}{*}{$\begin{array}{l}\text { Independent } \\
\text { Variable }\end{array}$} & \multicolumn{6}{|c|}{ Comfortable Gait Speed } \\
\hline & $R^{2}$ & $R^{2}$ Change & $\begin{array}{c}\text { Adjusted } \\
R^{2}\end{array}$ & $\begin{array}{c}\text { Unstandardized } \\
\text { B (Standard } \\
\text { Error) }\end{array}$ & $\begin{array}{c}\text { Standardized } \\
\beta\end{array}$ & $P$ \\
\hline Model 1 & 0.465 & 0.465 & 0.448 & & & \\
\hline $30-S C S$ & & & & $1.98(0.4)$ & 0.47 & $<.001$ \\
\hline FR & & & & $4.68(1.06)$ & 0.42 & $<.001$ \\
\hline Model 2 & 0.518 & 0.053 & 0.486 & & & \\
\hline $30-S C S$ & & & & $1.86(0.4)$ & 0.44 & $<.001$ \\
\hline FR & & & & $3.37(1.16)$ & 0.3 & 0.005 \\
\hline DSST completed & & & & $0.35(0.22)$ & 0.16 & 0.118 \\
\hline Flanker Effect & & & & $-0.02(0.01)$ & -0.19 & 0.046 \\
\hline
\end{tabular}

Notes: 30-SCS, 30-Second Chair Stand; FR, Functional Reach; DSST, Digit Symbol Substitution Test

Table 4 Hierarchical linear regression summary for fast gait speed $(\mathrm{cm} / \mathrm{s})$.

\begin{tabular}{|c|c|c|c|c|c|c|}
\hline \multirow[b]{2}{*}{$\begin{array}{c}\text { Independent } \\
\text { Variable }\end{array}$} & \multicolumn{6}{|c|}{ Fast Gait Speed } \\
\hline & $R^{2}$ & $R^{2}$ Change & $\begin{array}{c}\text { Adjusted } \\
R^{2}\end{array}$ & $\begin{array}{c}\text { Unstandardized } \\
\text { B (Standard } \\
\text { Error) }\end{array}$ & $\begin{array}{c}\text { Standardized } \\
\beta\end{array}$ & $P$ \\
\hline Model 1 & 0.401 & 0.401 & 0.382 & & & \\
\hline $30-S C S$ & & & & $2.9(0.62)$ & 0.46 & $<.001$ \\
\hline FR & & & & $5.64(1.59)$ & 0.35 & 0.001 \\
\hline Model 2 & 0.468 & 0.066 & 0.433 & & & \\
\hline $30-S C S$ & & & & $2.61(0.61)$ & 0.42 & $<.001$ \\
\hline FR & & & & $3.58(1.73)$ & 0.22 & 0.042 \\
\hline DSST completed & & & & $0.95(0.36)$ & 0.32 & 0.011 \\
\hline CDT Score & & & & $0.81(1.78)$ & 0.05 & 0.651 \\
\hline
\end{tabular}

Notes: 30-SCS, 30-Second Chair Stand; FR, Functional Reach; DSST, Digit Symbol Substitution Test; CDT, Clock-draw test

\section{Discussion}

The results of this study contribute to the current literature of predictors of comfortable and fast gait speed in community-dwelling older adults. It investigated the interplay of physical performance measures and specific cognitive domains that have previously been identified separately in the existing literature as variables associated with gait speed. Results indicate that lower extremity strength, balance, and executive function were unique predictors of comfortable gait speed while lower extremity strength, balance, and processing speed were unique predictors of fast gait speed. As summarized in the hierarchical linear regression model 2, these predictors explained $48.60 \%$ of the total variance in comfortable gait speed and $43.30 \%$ of the total variance 
in fast gait speed. The results support the first hypothesis, that strength and balance would both be strong predictors of comfortable and fast gait speed, even after the addition of the cognitive variables. The second hypothesis was partially supported. Executive function was found to be the strongest cognitive predictor of comfortable gait speed but not fast gait speed.

These results corroborate previous research findings that lower extremity strength and balance are key predictors of gait speed. These findings add to the body of literature by providing evidence that both are predictive for both comfortable and fast gait speed. The relationship between lower extremity strength and gait speed has been demonstrated primarily using measures of dynamometry [14, 18, 27, 28]. Specifically, knee extensor, hip extensor, and ankle plantarflexor strength have all been shown to have associations with gait speed using these methods $[18,28]$. In a recent study by the Mantel et al. (2018), the 30-SCS demonstrated significant predictive value for comfortable and fast gait speed. The action of rising from a chair in the 30-SCS requires the activation hip and knee extensors, allowing practicing clinicians to perform a functional assessment of lower extremity strength when gait speed deficits are present. The effect of balance on gait speed has been less studied and the available literature lacks homogeneity of balance assessments. Studies have shown positive associations between gait speed and measures of postural sway, sensory integration, and limits of stability [14-17]. While these results are encouraging, future research is needed to determine which components of balance are most influential to gait speed for specificity of intervention development.

Analyses revealed that the addition of cognitive variables showed significant contributions to the total variance in both comfortable and fast gait speeds. This contribution remained significant in spite of the large amount of variance explained by strength and balance. This additional variance explained adds to the literature by highlighting the interplay of physical and cognitive variables affecting the outcome of gait speed. Both physical and cognitive factors have a role and thus are important to consider during an exam.

In a 2016 meta-analysis of 26 cross-sectional studies investigating the relationship between gait and cognition, authors found small effect sizes in favor of positive association between gait speed and executive function and processing speed [48]. In agreement with previous cross-sectional findings $[20,21]$, this study found associations between comfortable gait speed and cognitive domains of executive function and processing speed. However, few studies have included fast gait speed when investigating the association between gait speed and cognition [30, 31]. This study found differences in predictors, in that executive function independently predicted comfortable gait speed while processing speed independently predicted fast gait speed. This partially supports the study hypotheses that the executive function domain would be a unique predictor of both comfortable and fast gait speed. Soumare and colleagues (2009) found that processing speed was more specifically associated with fast gait speed than executive function after controlling for confounders. In contrast, another study found that executive function was independently associated with fast gait speed, but not comfortable gait speed, after adjusting for cofounders [31] These mixed findings highlight the complexity of cognitive processes' that are required to adapt to a less-automatic physical performance task such as fast walking. Multiple studies have demonstrated the influence of higher cognitive processes on gait under varying conditions such as fast walking or dual-task walking $[21,49,50]$. Additionally, these differences may be explained by the heterogeneity among studies investigating cognition and gait speed, with variations and overlap in the interpretation of the cognitive domain measured. In contrast to previous studies 
investigating cognition and gait speed, the current study also included physical performance variables which may have contributed to the non-significance of some cognitive measures in the final regression model, despite significance found in bivariate correlations.

The interplay found between physical performance and cognition urges clinicians and researchers to investigate and evaluate gait speed with an interdisciplinary approach. As stated previously, gait speed has been recognized as a useful screening tool to offer insight into future health status functional decline, and fall risk [5]. This study suggests that if an older adult was screened using a gait speed assessment and was found to have gait speed deficits, further assessment of both physical and cognitive function is warranted to identify the most appropriate therapeutic interventions. A geriatric patient is often under the care of multiple healthcare providers who have the ability to collaborate to optimize the health and functioning of an older adult. The simplicity of administering a gait speed assessment allows many healthcare professionals to perform a screening and evaluate the need for further assessment of gait speed predictors identified in current literature. Physical impairments of strength and balance may be evaluated and treated by a professional such as a physical therapist, while cognitive impairments are better served by a professional such as a physician or speech language pathologist.

This study is not without limitations. The sample was very homogenous, limiting the generalizability of the findings. Additionally, while the sample size was adequate to address the particular research question regarding the interplay between physical performance and cognition on gait speed, a larger sample would allow sub-analyses to be performed based on gender or age to determine any impact those variables may have on these findings as these variables may have some confounding effect on the interplay between cognitive and physical performance variables. Additionally, a larger sample size would allow more sophisticated statistics, such as structural equation modeling, to allow a better understanding of the relationships that have been identified in this study.

\section{Conclusions}

This study suggests that both comfortable and fast gait speed are primarily predicted by strength and balance. Many clinicians are qualified to use gait speed as a screening tool for older adults. When a gait speed deficit is found, commonly used clinical measures of functional lower extremity strength and balance can be used to further assess the source of the impairment. The interplay found between physical performance and cognition emphasizes the importance of interdisciplinary care. Future studies should investigate the longitudinal associations between gait speed, cognition, and physical performance variables to better understand the directionality of these relationships.

\section{Author Contributions}

Ashleigh Trapuzzano was involved in project conceptualization, project design, participant recruitment, data collection, interpretation of the results, and dissemination including writing this manuscript.

Sara Chizmar was involved in project conceptualization, project design, participant recruitment, data collection, interpretation of the results, and dissemination including writing this manuscript. 
Lauren Haffke was involved in project conceptualization, project design, participant recruitment, data collection, interpretation of the results, and dissemination including writing this manuscript.

Nicole Dawson is the Director of the Innovative Mobility Initiative (IMOVE) Lab at the University of Central Florida and was the senior researcher on the project. She oversaw all aspects of the project and completed the statistical analysis.

\section{Competing Interests}

The authors have declared that no competing interests exist.

\section{References}

1. United Nations. Ageing. Available from: http://www.un.org/en/sections/issuesdepth/ageing/.

2. The United States Census Bureau. The Nation's Older Population Is Still Growing 2017 [Available from: https://www.census.gov/newsroom/press-releases/2017/cb17-100.html.

3. Peel NM, Kuys SS, Klein K. Gait speed as a measure in geriatric assessment in clinical settings: A systematic review. J Gerontol A Biol Sci Med Sci. 2013; 68: 39-46.

4. Goldberg A, Schepens S. Measurement error and minimum detectable change in 4-meter gait speed in older adults. Aging Clin Exp Res. 2011; 23: 406-412.

5. Fritz S, Lusardi M. White paper:"walking speed: The sixth vital sign”. J Geriatr Phys Ther. 2009; 32: 2-5.

6. Cesari M, Kritchevsky SB, Penninx BW, Nicklas BJ, Simonsick EM, Newman AB, et al. Prognostic value of usual gait speed in well-functioning older people-results from the health, aging and body composition study. J Am Geriatr Soc. 2005; 53: 1675-1680.

7. Studenski S, Perera S, Patel K, Rosano C, Faulkner K, Inzitari M, et al. Gait speed and survival in older adults. JAMA. 2011; 305: 50-58.

8. Verghese J, Wang C, Holtzer R. Relationship of clinic-based gait speed measurement to limitations in community-based activities in older adults. Arch Phys Med Rehabil. 2011; 92: 844-846.

9. Guralnik JM, Ferrucci L, Pieper CF, Leveille SG, Markides KS, Ostir GV, et al. Lower extremity function and subsequent disability: Consistency across studies, predictive models, and value of gait speed alone compared with the short physical performance battery. J Gerontol A Biol Sci Med Sci. 2000; 55: M221-M231.

10. Artaud F, Singh-Manoux A, Dugravot A, Tzourio C, Elbaz A. Decline in fast gait speed as a predictor of disability in older adults. J Am Geriatr Soc. 2015; 63: 1129-1136.

11. Van Kan GA, Rolland Y, Andrieu S, Bauer J, Beauchet O, Bonnefoy M, et al. Gait speed at usual pace as a predictor of adverse outcomes in community-dwelling older people an International Academy on Nutrition and Aging (IANA) Task Force. J Nutr Health Aging. 2009; 13: 881-889.

12. Callisaya ML, Blizzard L, McGinley JL, Srikanth V. Risk of falls in older people during fastwalking-the TASCOG study. Gait Posture. 2012; 36: 510-515.

13. Best JR, Liu-Ambrose T, Boudreau RM, Ayonayon HN, Satterfield S, Simonsick EM, et al. An evaluation of the longitudinal, bidirectional associations between gait speed and cognition in older women and men. J Gerontol A Biol Sci Med Sci. 2016; 71: 1616-1623. 
14. Aranda-García S, Busquets A, Planas A, Prat-Subirana JA, Angulo-Barroso RM. Strength, static balance, physical activity, and age predict maximal gait speed in healthy older adults from a rural community: A cross-sectional study. J Aging Phys Act. 2015; 23: 580-587.

15. Mantel A, Trapuzzano A, Chizmar S, Haffke L, Dawson N. An investigation of the predictors of comfortable and fast gait speed in community-dwelling older adults. J Geriatr Phys Ther. 2019; 42: E62-E68.

16. Xie YJ, Liu EY, Anson ER, Agrawal Y. Age-related imbalance is associated with slower walking speed: An analysis from the National Health and nutrition examination survey. J Geriatr Phys Ther. 2017; 40: 183-189.

17. Callisaya ML, Blizzard L, Schmidt MD, McGinley JL, Lord SR, Srikanth VK. A population-based study of sensorimotor factors affecting gait in older people. Age Ageing. 2009; 38: 290-295.

18. Bean JF, Leveille SG, Kiely DK, Bandinelli S, Guralnik JM, Ferrucci L. A comparison of leg power and leg strength within the InCHIANTI study: Which influences mobility more? J Gerontol A Biol Sci Med Sci. 2003; 58: M728-M733.

19. Hicks GE, Shardell M, Alley DE, Miller RR, Bandinelli S, Guralnik J, et al. Absolute strength and loss of strength as predictors of mobility decline in older adults: The InCHIANTI study. J Gerontol A Biol Sci Med Sci. 2011; 67: 66-73.

20. Martin KL, Blizzard L, Wood AG, Srikanth V, Thomson R, Sanders LM, et al. Cognitive function, gait, and gait variability in older people: A population-based study. J Gerontol A Biol Sci Med Sci. 2012; 68: 726-732.

21. Killane I, Donoghue OA, Savva GM, Cronin H, Kenny RA, Reilly RB. Relative association of processing speed, short-term memory and sustained attention with task on gait speed: $A$ study of community-dwelling people 50 years and older. J Gerontol A Biol Sci Med Sci. 2014; 69: 1407-1414.

22. Atkinson HH, Rosano C, Simonsick EM, Williamson JD, Davis C, Ambrosius WT, et al. Cognitive function, gait speed decline, and comorbidities: The health, aging and body composition study. J Gerontol A Biol Sci Med Sci. 2007; 62: 844-850.

23. Miljkovic N, Lim JY, Miljkovic I, Frontera WR. Aging of skeletal muscle fibers. Ann Rehabil Med. 2015; 39: 155-162.

24. Goodpaster BH, Park SW, Harris TB, Kritchevsky SB, Nevitt M, Schwartz AV, et al. The loss of skeletal muscle strength, mass, and quality in older adults: The health, aging and body composition study. J Gerontol A Biol Sci Med Sci. 2006; 61: 1059-1064.

25. Cawthon PM, Fox KM, Gandra SR, Delmonico MJ, Chiou CF, Anthony MS, et al. Do muscle mass, muscle density, strength, and physical function similarly influence risk of hospitalization in older adults? J Am Geriatr Soc. 2009; 57: 1411-1419.

26. Buchner DM, Larson EB, Wagner EH, Koepsell TD, DE LATEUR BJ. Evidence for a non-linear relationship between leg strength and gait speed. Age Ageing. 1996; 25: 386-391.

27. Mangione KK, Craik RL, Lopopolo R, Tomlinson JD, Brenneman SK. Predictors of gait speed in patients after hip fracture. Physiother Can. 2008; 60: 10-18.

28. Muehlbauer T, Granacher U, Borde R, Hortobágyi T. Non-discriminant relationships between leg muscle strength, mass and gait performance in healthy young and old adults. Gerontology. 2018; 64: 11-18.

29. Cohen JA, Verghese J, Zwerling JL. Cognition and gait in older people. Maturitas. 2016; 93: 7377. 
30. Soumaré A, Tavernier B, Alpérovitch A, Tzourio C, Elbaz A. A cross-sectional and longitudinal study of the relationship between walking speed and cognitive function in communitydwelling elderly people. J Gerontol A Biol Sci Med Sci. 2009; 64: 1058-1065.

31. Ble A, Volpato S, Zuliani G, Guralnik JM, Bandinelli S, Lauretani F, et al. Executive function correlates with walking speed in older persons: The InCHIANTI study. J Am Geriatr Soc. 2005; 53: 410-415.

32. Holtzer R, Verghese J, Xue X, Lipton RB. Cognitive processes related to gait velocity: Results from the Einstein Aging Study. Neuropsychology. 2006; 20: 215.

33. Callisaya ML, Blizzard CL, Wood AG, Thrift AG, Wardill T, Srikanth VK. Longitudinal relationships between cognitive decline and gait slowing: The Tasmanian Study of Cognition and Gait. J Gerontol A Biol Sci Med Sci. 2015; 70: 1226-1232.

34. Watson N, Rosano C, Boudreau R, Simonsick E, Ferrucci L, Sutton-Tyrrell K, et al. Executive function, memory, and gait speed decline in well-functioning older adults. J Gerontol A Biol Sci Med Sci. 2010; 65: 1093-1100.

35. Cohen J. A power primer. Psychol Bull. 1992; 112: 155.

36. Rikli RE, Jones CJ. Development and validation of a functional fitness test for communityresiding older adults. J Aging Phys Act. 1999; 7: 129-161.

37. Duncan PW, Weiner DK, Chandler J, Studenski S. Functional reach: A new clinical measure of balance. J Gerontol. 1990; 45: M192-M197.

38. Juby A, Tench S, Baker V. The value of clock drawing in identifying executive cognitive dysfunction in people with a normal Mini-Mental State Examination score. Can Med Assoc J. 2002; 167: 859-864.

39. Arbuthnott K, Frank J. Trail making test, part B as a measure of executive control: Validation using a set-switching paradigm. J Clin Exp Neuropsychol. 2000; 22: 518-528.

40. Eriksen BA, Eriksen CW. Effects of noise letters upon the identification of a target letter in a nonsearch task. Percept Psychophys. 1974; 16: 143-149.

41. Kaufman AS. Test Review: Wechsler, D. Manual for the wechsler adult intelligence scale, revised. New York: Psychological Corporation, 1981. J Psychoeduc Assess. 1983; 1: 309-313.

42. GAITRite. GAITRite Electronic Walkway Technical Reference. Franklin, NJ: CIR Systems, Inc; 2016. Available from: http://gaitrite.legacy.si-servers.com/WI-02-

15 Technical Reference T.pdf.

43. Watson $\mathrm{YI}$, Arfken CL, Birge SJ. Clock completion: An objective screening test for dementia. J Am Geriatr Soc. 1993; 41: 1235-1240.

44. Neurobehavioral Systems. Psych Lab 101. 2.02 ed. Berkeley, CA: Neurobehavioral Systems, Inc; 2015.

45. Holtzer R, Mahoney J, Verghese J. Intraindividual variability in executive functions but not speed of processing or conflict resolution predicts performance differences in gait speed in older adults. J Gerontol A Biol Sci Med Sci. 2013; 69: 980-986.

46. Bobko P. Correlation and regression: Applications for industrial organizational psychology and management. Sage; 2001.

47. Tabachnick BG, Fidell LS, Ullman JB. Using multivariate statistics. Pearson Boston, MA; 2007.

48. Demnitz $N$, Esser $P$, Dawes $H$, Valkanova $V$, Johansen-Berg $H$, Ebmeier KP, et al. A systematic review and meta-analysis of cross-sectional studies examining the relationship between mobility and cognition in healthy older adults. Gait Posture. 2016; 50: 164-174. 
49. Lowry KA, Brach JS, Nebes RD, Studenski SA, VanSwearingen JM. Contributions of cognitive function to straight-and curved-path walking in older adults. Arch Phys Med Rehabil. 2012; 93: 802-807.

50. Coppin AK, Shumway-Cook A, Saczynski JS, Patel KV, Ble A, Ferrucci L, et al. Association of executive function and performance of dual-task physical tests among older adults: Analyses from the InChianti study. Age Ageing. 2006; 35: 619-624.

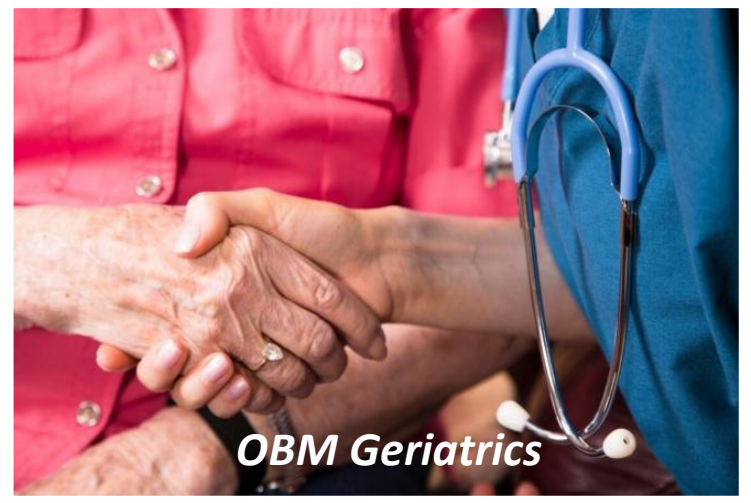

Enjoy OBM Geriatrics by:

1. Submitting a manuscript

2. Joining in volunteer reviewer bank

3. Joining Editorial Board

4. Guest editing a special issue

For more details, please visit:

http://www.lidsen.com/journals/geriatrics 